\title{
The importance of osmolality in hydrophilic urethral catheters: a crossover study
}

\author{
L Waller, M Telander and L Sullivan
}

Spinal Injuries Unit, Department of Neurosurgery, Sahlgrenska Hospital, Göteborg, Sweden

\begin{abstract}
The longterm use of clean intermittent self catheterisation as an option for bladder management in the care of spinal cord injury patients has shown the possible risk of urethral trauma and chronic infections being developed over time. The basic properties of the catheters being used need to be evaluated. In this crossover study of 14 male spinal cord injury patients, two commercial hydrophilic catheters were compared, as to the maximum friction force during the removal of the catheters after bladder emptying. The friction force, measured by a dynamometer twice daily, showed significant lower values for LoFric $(0.87 / 0.84 \mathrm{~N})$ than for EsiCath/Conveen $(1.38 / 1.27 \mathrm{~N})$. Sticking to the urethral epithelium was reported three times (two patients) with the LoFric catheter, and 42 times (nine patients) with the EasiCath catheter. Osmolality of the outer layer of the catheters was measured using a freezing-point reduction technique. The greater than 10 times higher osmolality $(\sim 900 \mathrm{mOsm} / \mathrm{kg})$ of the Lofric catheter may explain the results.
\end{abstract}

Keywords: spinal cord injuries; urethral catheterisation; osmolality of catheters

\section{Introduction}

Intermittent catheterisation (IC) and clean intermittent catheterisation (CIC) are the results of innovative ideas which have been most prevalent in the medical health and psycho-social life of spinal injury patients during the past 40 years. ${ }^{1-5}$ People's doubts surrounding new paradigms should be seen more as a result of tradition than a lack of technology for running conclusive, comparative studies. Nowadays, the technology exists but a decisive routine for starting randomised clinical trials is lacking in more or less the entire spinal injury area. Within the field of neurology, detailed long-term follow-ups of the Lapides method have stated that urethral complications and chronic infections are dependent on the length of the regimen, ${ }^{6-9}$ while the hydrophilic CIC catheters could reduce these complications. ${ }^{0,11}$ The time seems right for a comparative prospective clinical trial. ${ }^{9}$

The hydrophilic catheter which has so far been tested (LoFric) seems to cause less short-term strains ${ }^{12}$ and long-term trauma ${ }^{10,11}$ and should logically reduce the risk of chronic prostatitis and epididymitis.,10 Positive results of self-catheterisaton have also been seen in stricture treatment following urethrotomy ${ }^{13}$ including the low-friction variation. ${ }^{14}$ Conclusive support for this requires prospective and sufficiently large-scale studies over many years, where the optimal properties for the atraumatic performance of the catheters involved are tested and compared.

Correspondence: L Waller, Eklandagatan 52 C, S-412 61, Göteborg, Sweden
This preparatory clinical study in a crossover design compares the friction during catheter removal between two commercial hydrophilic catheters as part of the spinal injury patient's usual bladder regimen during the post-injury phase.

\section{Background}

The Spinal Injuries Unit at Sahlgrenska Hospital, regionally responsible for the development of health care and methods within the spinal injury area, was asked in 1983, to conduct clinical tests of a new urethral catheter, LoFric, which at that time represented a new hydrophilic principle for intermittent and clean intermittent catheterisation. The catheter was described as a sterile plastic (polyvinyl chloride) catheter coated with a non-reactive hydrophilic polymer (polyvinyl pyrrolidone) which could bind lubricating liquids and reduce friction during catheterisation. It was recommended for single use only. In vitro studies had shown that the catheter had 10-15 times lower friction than the regular plastic catheter with gel. ${ }^{15}$

We thought that suitable SCI patients should try to use a hydrophilic low-friction catheter, because logically it should reduce urethral trauma with longterm use and lead to a small but important revision of the traditional use of catheters using gel as lubricator. An ambitious crossover study was initiated for a random comparison of the LoFric catheter with an ordinary catheter with gel from the acute injury to 5 
years post-injury. However, due to the high drop-out rate following crossover from the LoFric catheter after 3 months' usage, this study could not be completed. The failure was reported to the International Medical Society of Paraplegia in 1985, with an appeal for participation in a multi-centre study, but this attracted little response. However, approximately 1000 catheters were tested at the Spinal Injuries Unit at the Royal Perth Hospital in Western Australia in the autumn of 1985. After 6 months, it was reported that the catheter worked well, but the neurology consultant reported that the catheter had shown a tendency to stick to the urethra in a few patients, which could have serious consequences (private correspondence from Sir George Bedbrook, 1985). A small number of similar incidents also occurred in our unfinished study with patients who, after being discharged from hospital, used tap water as a lubricant. Also, as has been reported, one patient had advanced urethral damage in the acute phase. ${ }^{11}$

The manufacturer reviewed the hydrophilisation process and produced a second generation of LoFric (first named Salt LoFric) with sodium chloride in the outer layer which, when soaked in water, increased osmolality from $\sim 30 \mathrm{mOsm} / \mathrm{kg}$ to $\sim 800 \mathrm{mOsm} / \mathrm{kg}$ as in human urine. ${ }^{16}$

The catheter was tested in a random study on dogs which showed marked epithelial damage of the urethral mucosa after a catheterisation regimen using a polyvinyl chloride catheter with chlorhexidine gel as lubricator, compared to discrete local hyperaemia and mild superficial reactions after using the hydrophylic catheters with sodium chloride as lubricator. The traumatic effect of the tested catheters seemed to be highly dependent on the friction of the catheter. No evidence was apparently found for the impact of osmolality of the hydrophilic catheter. In the study the friction was equal for the two LoFric catheters. Catheterisation time was standardised to $60 \mathrm{s.}^{16}$

However, there still seems to be a logical connection between osmolality and friction. The theoretical background is that a catheter with the outer layer consisting of sodium chloride raises the osmolality of the catheter, and in turn its water binding ability, to ideal levels. The osmolality of a hydrophilic catheter should equalize the osmolality of human urine. Soaked in water, the polyvinyl pyrrolidone chains bind the lubricating liquid to approximately the same osmolality as exists in the urethral epithelium. There is therefore a great reduction in friction between the catheter and urethral mucosa because the contact area mainly consists of water molecules. The further development of the LoFric catheter has been to raise the osmolality even higher by adding more sodium chloride to the hydrophilic layer.

Following 3 years of clinical assessment in Stockholm and Gothenburg, the second generation of catheters was approved for free distribution in Sweden, ie it could be administered free to patients - this soon applied to all Scandinavian countries. There have since been no reports of serious adhesions to the urethra in any Scandinavian country. With this long experience of the LoFric catheter we were able to accomplish a long-term follow-up on patients using the hydrophilic catheters. ${ }^{11}$

Between 1990 and 1992, a number of catheter manufacturers asked us to carry out comparative studies between their catheters and the LoFric catheter, both with inpatients using saline solution as lubricator and with outpatients using tap water as lubricator. On several occasions, preparations for such studies showed that the alternative catheter had a tendency to stick to the urethra wall when using water as lubricator. This also occurred when preparing this study comparing the LoFric and EasiCath catheters using water as lubricator. Although our request for information concerning the osmolality of the hydrophilic layer was not respected by the manufacturer of the EasiCath, we decided to carry on with the test on our own initiative.

\section{Material and methods}

Fourteen consecutive male SCI patients treated at the Spinal Injuries Unit at Sahlgrenska Hospital from August to December 1994 took part in the study. The median age of the patients was 30 years (22 to 62 years). Prior to implementation of the study protocol, all patients used the LoFric catheter in accordance with the unit's routines from the day of injury. Post-injury time spanned from 5 days to 5 months. There were eight tetraplegic and five paraplegic patients resulting from trauma, and one patient had spinal paralysis from haemorrhage from a cervical vascular deformity (level C6-7). Eight patients had complete neurological injuries and six incomplete. Three patients had regained bladder function before the study began but the other patients were still in the shock phase. When the patients had regained bladder function they were scheduled for urological evaluation including cystometry, excretory urography, cystoscopy, chromEDTA clearance and radiorenography.

In this study, LoFric ${ }^{\mathrm{R}}\left(\mathrm{C}_{\mathrm{LF}}\right)$ (Astra Tech AB, Mölndal, Sweden), the hydrophilic catheter that has been used for a long time in the unit when performing intermittent catheterisation, was used as the control for another commercial hydrophilic catheter with unknown physical properties, EasiCath/Conveen $\left(\mathrm{C}_{\mathrm{EC}}\right)$ (Coloplast $\mathrm{A} / \mathrm{S}$, Espergærde, Denmark). The study was primarily designed for tap-water as lubricator. However, during an introductory period when catheterisation was timed and standardised, it was noted that the $\mathrm{C}_{\mathrm{EC}}$ was showing severe adhesion to urethra a few times. The bladders were filled with saline solution before the catheters could be carefully removed. After this it was decided to change the design of the study to the use of saline as lubricator.

Patients were randomly allocated to the use of one of the catheters for 10 days and then crossed over to the other for the same amount of time. The maximum friction during standardised removal of the catheter 
$(10 \mathrm{~cm} / \mathrm{sec})$ was measured with an electronic dynamometer (Mecmesin AFG-25) twice daily (morning and afternoon). During the study the patient was to use the same type of catheter for each catheterisation (4-5 times a day) for the 10 days. The same experienced nurse performed all of the catheterisations. The patients were not told which catheter was being used, as was a third person who read the dynamometer. However, this person was probably not completely 'blind' as the colour of the two catheters varied slightly. It was impossible to 'blind' the nurse performing the catheterisations due to the different characteristics of the two catheters when soaked into saline solution. Catheterisation ended with manual compression. The duration of the whole emptying procedure was measured and the catheter size used was registered.

Urine cultures are performed routinely twice weekly on all of the patients in the unit irrespective of their bladder regimen. Whilst in hospital, patients with clinical infections and those with asymptomatic bacteriuria but with urine cultures with $>10,000$ colonyforming units per $\mathrm{ml}$ urine receive antibacterial treatment according to resistance pattern. For this reason, five patients had been treated with antibiotics according to resistance pattern during the single month period before the study. No patient used any other medication which would affect the urinary tract and they were only allowed a fluid intake of $<2$ litres per day.

\section{Results}

All osmolality measurements of the hydrophilic outer layer were carried out using measurements of the reduction of the freezing point. Independent measurements were carried out at the Technical Research Centre in Finland, Tampere (Finland) and at the Astra Hässle laboratories, Mölndal (Sweden). Widespread samples of both catheters were brought in from different batches in Finland and Sweden.

The catheters were soaked in distilled water for 30 s. A hydrophilic surface sample of the catheters was diluted with distilled water 1:3 and 1:4 and three times measured in an Advanced Instrument Model $3 \mathrm{C} 2$ osmometer. A control value of $290 \mathrm{mOsm} / \mathrm{kg}$ was used. The measuring range of the instrument was $0-$ $2000 \mathrm{mOsm} / \mathrm{kg}$. All measurements for osmolality for $\mathrm{C}_{\mathrm{LF}}$ were confirmed as being in the area for human urine, $800-1150 \mathrm{mOsm} / \mathrm{kg}$, ${ }^{17}$ with a mean of $900 \mathrm{mOsm} / \mathrm{kg}$ for $\mathrm{C}_{\mathrm{LF}}$ in Tampere and $950 \mathrm{mOsm} /$ $\mathrm{kg}$ in Mölndal. The corresponding figures for $\mathrm{C}_{\mathrm{EC}}$ was $15 \mathrm{mOsm} / \mathrm{kg}$ in Tampere and $80 \mathrm{mOsm} / \mathrm{kg}$ in Mölndal. ${ }^{18,19}$

A total of 526 friction measurements were performed. The median values of the maximum removal friction, recorded twice daily, were $0.87 /$ $0.84 \mathrm{~N}$ with $\mathrm{C}_{\mathrm{LF}}(88.7 / 85.5$ grams) and $1.38 / 1.27 \mathrm{~N}$ with $\mathrm{C}_{\mathrm{EC}}(140.0 / 129.9$ grams), resulting in an overall difference between the catheters of $55 \%(P<0.001)$, measured as mean values per patient per day. The variation of measurements varied from 0.01 to $1.69 \mathrm{~N}$ for $\mathrm{C}_{\mathrm{LF}}$ and from 0.24 to $7.50 \mathrm{~N}$ for $\mathrm{C}_{\mathrm{EC}}$. The difference in favour of $\mathrm{C}_{\mathrm{LF}}$ was consistent throughout all the patients, with the exception of one patient. No measurement for $\mathrm{C}_{\mathrm{LF}}$ catheterisation exceeded $2.0 \mathrm{~N}$ whereas this occurred in 50 cases $(20.2 \%)$ in 12 patients during catheterisation with $\mathrm{C}_{\mathrm{EC}}$.

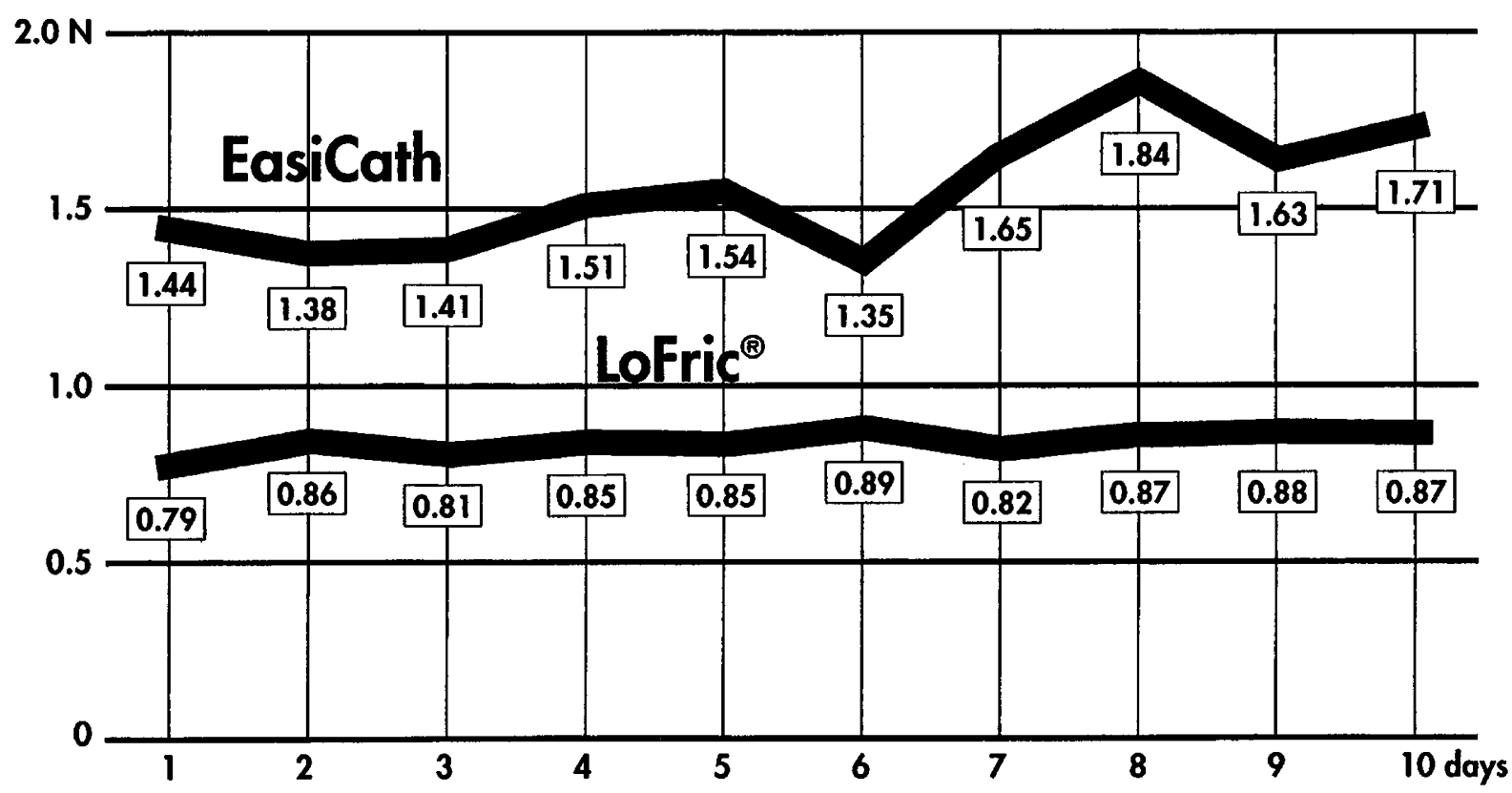

Figure 1 Mean values of the removal friction $(\mathrm{N})$ from all catheterisations assessed at day 1 to day 10 in 14 SCI male patients using LoFric and EasiCath catheters 
The performing nurse reported three stickings in two patients when removing the $C_{L F}$ as signs of adherence to the urethra. The same complication was reported 42 times in nine patients with $\mathrm{C}_{\mathrm{EC}}$. No haemorrhage was reported. Two episodes of urinary tract infections were registered during catheterisation with $\mathrm{C}_{\mathrm{LF}}$ and three with $\mathrm{C}_{\mathrm{EC}}$ but the catheterisations could continue according to the protocol without affecting the measurements. The five patients were treated with antibiotics according to the resistance pattern.

No influence on frictional force relating to level and completeness of injury, shock phase or final diagnosis of neuropathic bladder was observed. No relation was found between catheterisation time and friction. Mean catheterisation time was 4.3 min for $\mathrm{C}_{\mathrm{LF}}(3.5-8.2)$ and for $\mathrm{C}_{\mathrm{EC}} 4.4 \mathrm{~min}(3.3-9.6)$. The size of the catheter (F12 and F14) showed no impact on the friction. In a few patients, in the rehabilitation phase, it was also possible to perform measurements in different positions but body position did not seem to influence friction.

\section{Discussion}

The development of important clinical routines in the treatment and care of spinal cord injuries has, with few exceptions, taken place without scientifically-based prospective comparisons between new and old methods. This is also the case in the care of the neuropathic bladder. Health care's incentives to change established routines are of course small, unless new methods have proved to be more effective, safer and economically viable. Clinical trials are increasingly required for comparisons before extensive reorganisations of important routines are carried out. However, such routines are demanding for patients, complex, often difficult to carry out and sometimes take a very long time, so that the general medical requirements when the study is completed are clearly different from those at the start. The fact that clinically ideal studies on small groups as SCI patients are difficult and sometimes utopian explains why development is still powered by interpretations and comparisons between well-structured follow-up studies.

The difficulty in organising prospective, comparative intervention studies as a basis for clinical routines, creates a market for untested technical instruments and aids with poor documentation, e.g. hydrophilic urethral catheters. In the marketing of such catheters, documentation of the hydrophilic qualities which are decisive, such as the level of osmolality as a basis for clinical suitability, is often totally missing. Basic information, such as results from physical test programs and animal experiments, as well as clinical test results, is also missing. Manufacturers seem to think that referring to the hydrophilicity as a uniform concept, and to a well documented product already available in the market, like the LoFric catheter, is sufficient and that price is the most important decisive factor. Missing product information, basic test results and documentation as referred to above, have been promised, but never provided, by the manufacturers of new hydrophilic catheters, including the catheter tested against LoFric in this study.

No influence on frictional force relating to level and completeness of injury, shock phase, final diagnosis of the neuropathic bladder, catheterisation time or size of catheter being used was observed. Our own experience during the early stages of the development of the LoFric catheter and the result of this present study indicates the importance of the osmolality of the outer layer of hydrophilic catheters. As osmolality is a decisive factor for a hydrophilic catheter's water retention and, hence, level of friction, it may help to reduce the risk of catheter-induced microtrauma on the urethral epithelium. The higher osmolality of the LoFric catheter in this study may explain the lower rate of adhesion and potential damage with this catheter. Presumably, the outer layer should possess the same osmolality as the urine which passes the urethral epithelium when the bladder is emptied. Patient safety demands knowledge of the properties of the hydrophilic catheter before use. It is also important that these properties are carefully defined before a large-scale clinical study is carried out as is proposed.

\section{References}

1 Guttmann L, Frankel H. The value of intermittent catheterisation in the early management of traumatic paraplegia and tetraplegia. Paraplegia 1966; 4: 63-84.

2 Lapides J, Diokno AC, Silber SJ, Lowe BS. Clean, intermittent self-catheterization in the treatment of urinary tract disease. $J$ Urol 1972; 107: 458-461.

3 Lapides J, Diokno AC, Lowe BS, Kalish MD. Followup on unsterile, intermittent self-catheterization. J Urol 1974; 111: $184-187$.

4 Lapides J, Diokno AC, Gould FR, Lowe BS. Further observations in self-catheterization. J Urol 1976; 116: 169-171.

5 McGuire EJ, Savastano JA. Long-term followup of spinal cord injury patients managed by intermittent catheterization. J Urol 1983; 129: $775-776$.

6 Labat JJ et al. L'autosondage intermittent propre dans la réeducation des blessés médullaires et de la queue de cheval. I et II. Ann Réadaptation Med Phys 1985; 28: $111-123,125-136$.

7 Wyndaele JJ, Maes D. Clean intermittent self-catheterization: A 12 year followup. J Urol 1990; 143: 906-908.

8 Perkash I, Giroux J. Clean intermittent catheterization in spinal cord injury patients: A followup study. J Urol 1993; 149: 1068 1071.

9 Perrouin-Verbe B et al. Clean intermittent catheterisation from the acute period in spinal cord injury patients. Long term evaluation of urethral and genital tolerance. Paraplegia 1995; 33: $619-624$.

10 Bakke A, Vollset SE, Hoisaeter PA, Irgens LM. Physical complications in patients treated with clean intermittent catheterization. Scand J Urol Nephrol 1993; 27: 55-61.

11 Waller L, Jonsson O, Norlén L, Sullivan L. Clean intermittent catheterization in spinal cord injury patients: Long-term followup of a hydrophilic low-friction technique. J Urol 1995; 153: $345-348$.

12 Vaidyanathan S, Soni BM, Dundas S, Krishnan KR. Urethral cytology in spinal cord injury patients performing intermittent catheterisation. Paraplegia 1994; 32: 493 - 500. 
13 Newman LH, Stone NN, Chircus JH, Kramer HC. Recurrent urethral stricture disease managed by clean intermittent selfcatheterization. J Urol 1990; 144: $1142-1143$.

14 Lawrence WT, MacDonagh RP. Treatment of urethral stricture disease by internal urethrotomy followed by intermittent 'lowfriction' self-catheterization: preliminary communication. $J$ Roy Soc Med 1988; 81: $136-139$

15 Brattö M, Nordin K. Friktionsmätning. The Swedish National Testing and Research Institute, Report 8321, 1018, Borås, Sweden 1983

16 Carlsten J, Bowall S, Sullivan L, Nilsson A. Effect of catheterization and surface osmolarity on urethral epithelium. An experimental study on dogs. Read at the Int Med Soc Paraplegia Annual Scientific Meeting, Stoke Mandeville Hospital, Aylesbury, England, 1987.
17 Dunger DB et al. A short water deprivation test incorporating urinary arginine vasopressin estimations for the investigation of posterior pituitary function in children. Acta Endocr 1988; 117: $13-18$.

18 Astra Tech AB, Mölndal, Sweden (On file with that laboratory).

19 Technical Research Centre of Finland. Research report no KET/ 3229/95, Tampere, Finland 1995. 